\title{
Platelet Rich Fibrin in Periodontal Practice, Review
}

\author{
Abdullah Saleh Almutairi* \\ Department of Periodontics and Oral Medicine, College of Dentistry, Qassim University, KSA \\ *Corresponding author: Dr.almutairi@qu.edu.sa
}

\begin{abstract}
Autologous platelet concentrates have become a rage in the field of regenerative medicine including numerous specialties like orthopedics, maxillofacial and cosmetic surgery, sports medicine and dentistry. The use of these platelet concentrates ranging from fibrin glues to the currently used platelet-rich fibrin (PRF) has exponentially increased and it has brought about a radical change in the stimulation and acceleration of regenerative tissue processes. The current review highlights the evolution of different platelet concentrates and the use of platelet rich fibrin in periodontal treatment and tissue repair.
\end{abstract}

Keywords: platelet rich concentrates, platelet rich fibrin, periodontics, regeneration, tissue repair

Cite This Article: Abdullah Saleh Almutairi, "Platelet Rich Fibrin in Periodontal Practice, Review." International Journal of Dental Sciences and Research, vol. 5, no. 5 (2017): 132-136. doi: 10.12691/ijdsr-5-5-4.

\section{Introduction}

Fibrin is the activated form of plasmatic molecule (fibrinogen) [1]. Fibrinogen is a soluble protein and it gets transformed into insoluble fibrin by the enzyme known as thrombin. The resultant polymerized fibrin gel constitutes the first cicatricial matrix of the injured site. This soluble fibrillary molecule is massively present both in plasma and in the platelet granules and plays a pivotal role in platelet aggregation during hemostasis. The biochemical process involves its transformation into a biologic glue capable of consolidating the initial platelet cluster, thus constituting a protective wall along vascular breaches during coagulation. Fibrinogen remains the final substrate in all coagulation reactions $[2,3]$.

\subsection{Fibrin Adhesives}

Fibrin adhesives were commonly used due to its healing properties, including potential to accelerate healing and reducing the formation of postoperative hematoma. [4] The shortcomings of this adhesive system were that it had extremely long and complex protocols, clerical error by the blood bank increasing the risk of the patient towards adverse transfusion reaction or infectious diseases in addition to patient's need to adhere to the blood bank's criteria for age, weight, hemoglobin concentration and health status [5]. Since it was too hard to prepare, these techniques were not widely developed.

\subsection{Platelet-Rich Plasma}

Platelet rich plasma (PRP) is a modification of the fibrin adhesive that requires autologous blood collection in the immediate preoperative period. It is an active concentrate of platelets which makes up to $95 \%$ of its content, $4 \%$ red blood cells and $1 \%$ white blood cells. It triggers a cascade of growth factors such as platelet-derived growth factor (PDGF), transforming growth factor- $\beta$ (TGF- $\beta$ ), insulin-like growth factor (IGF-1) and platelet factor 4(PF-4). It also contains proteins like fibrin, fibronectin and vitronectin which act as adhesion molecules for osteoconduction and epithelial migration. The processing is carried out using a centrifugation machine using variable speed salvage system and is then mixed with bovine thrombin and calcium chloride. This causes the gelling and activation of the platelet concentrate [6].

There are various classification systems for these platelet concentrates, but the recently developed system has categorized into 4 broad families based on the leukocyte content and fibrin architecture [7].

a. Pure platelet-rich plasma (P-PRP)-Liquid suspension without leukocytes prior to activation (Ex: Vivostat PRF, Anitua's PRGF). Can be transformed to P-PRP gel.

b. Leukocyte and Platelet-rich plasma (L-PRP)-Liquid suspension with leukocytes before activation. (Ex: Curasan Regen, Smartprep, Magellan). Can be activated and transformed to L-PRP gel.

c. Pure Platelet-rich fibrin (P-PRF)-Solid fibrin material without leukocytes (Ex: Fibrinet)

d. Leukocyte and Platelet-rich fibrin (L-PRF)-Solid fibrin material with leukocytes (Ex: Choukroun's PRF, Advanced PRF).

\subsection{Platelet-Rich Fibrin}

It is a second-generation platelet concentrate which was first developed by Choukroun in 2001 [8]. This generation of platelet concentrates alleviates the use of calcium chloride or bovine thrombin for gelling and activation. Thus, the risk of developing antibodies with the use of bovine thrombin against factors $\mathrm{V}, \mathrm{XI}$ and thrombin is negotiated in PRF. 


\subsection{Preparation of Platelet-Rich Fibrin}

The preparation of PRF requires PC-02 table centrifuge and a blood collection kit. This follows the French technique as proposed by Choukroun [8]. This process involves simple protocol where the collected blood from the patient is immediately centrifuged to obtain the PRF clot. Since there are no anticoagulants added, the protocol warrants swiftness which counters the clotting cascade of the blood when in contact with the test tube. In the end, a fibrin clot super charged with platelets and serum is obtained.

The desired quantity of venous blood (around $5 \mathrm{ml}$ ) is collected in each of the two sterile vacutainer tubes of 6 $\mathrm{ml}$ capacity without anticoagulant. The vacutainer tubes are then placed in a centrifugal machine at 3000 revolutions per minute (rpm) for 10 minute (36). The blood gets separated into three layers [Figure 1]:

1. Uppermost layer containing acellular platelet-poor plasma

2. Middle layer containing the PRF clot

3. Bottom layer consisting of the RBC's.

The autologous fibrin membranes can be obtained by squeezing out the serum from the clot [Figure 2]. The blood begins to coagulate when it comes in contact with the glass surface of the test tube, so faster blood collection and immediate centrifugation are the key factors in delaying the clotting cascade and for the successful formation of PRF [9].

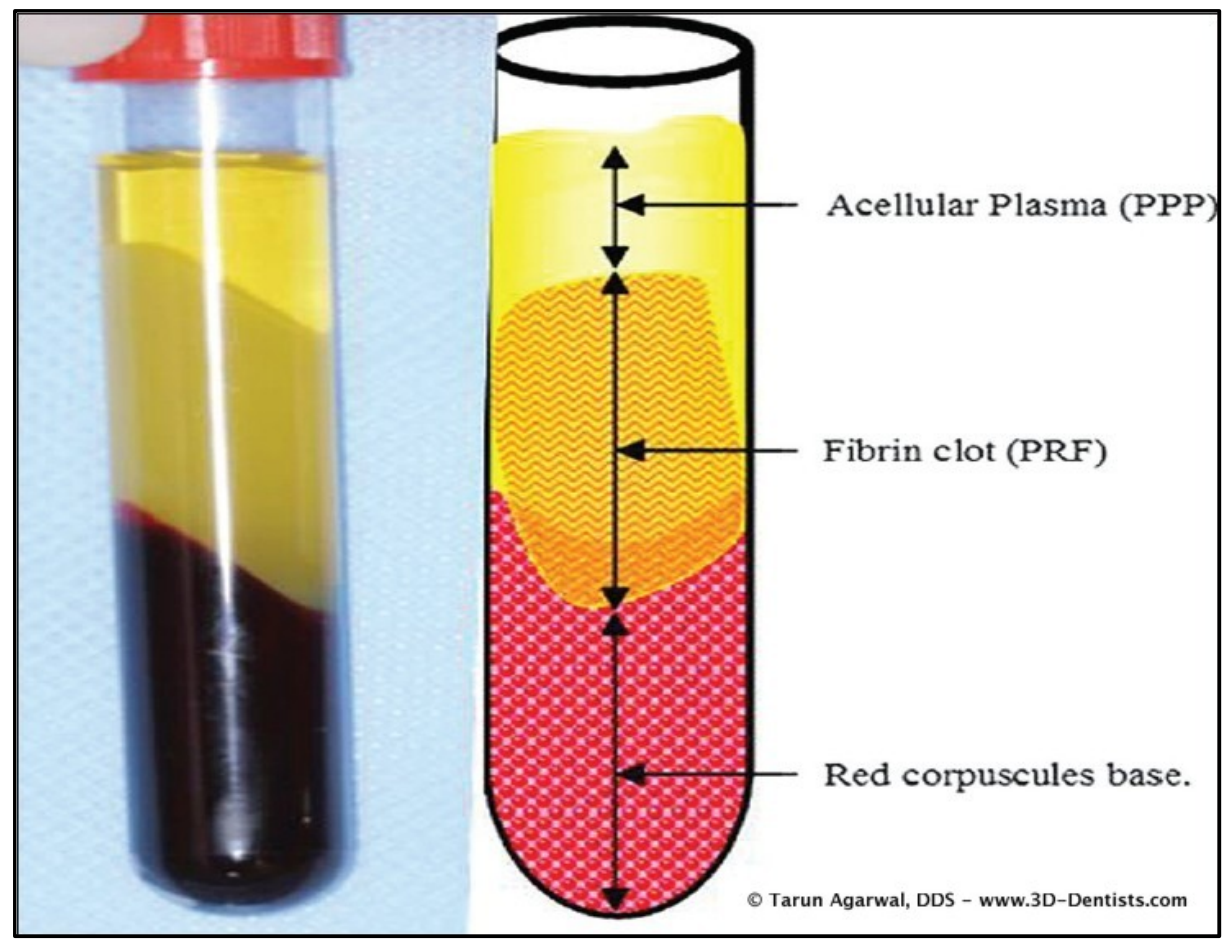

Figure 1. Preparation of Platelet-rich fibrin- 3 layers

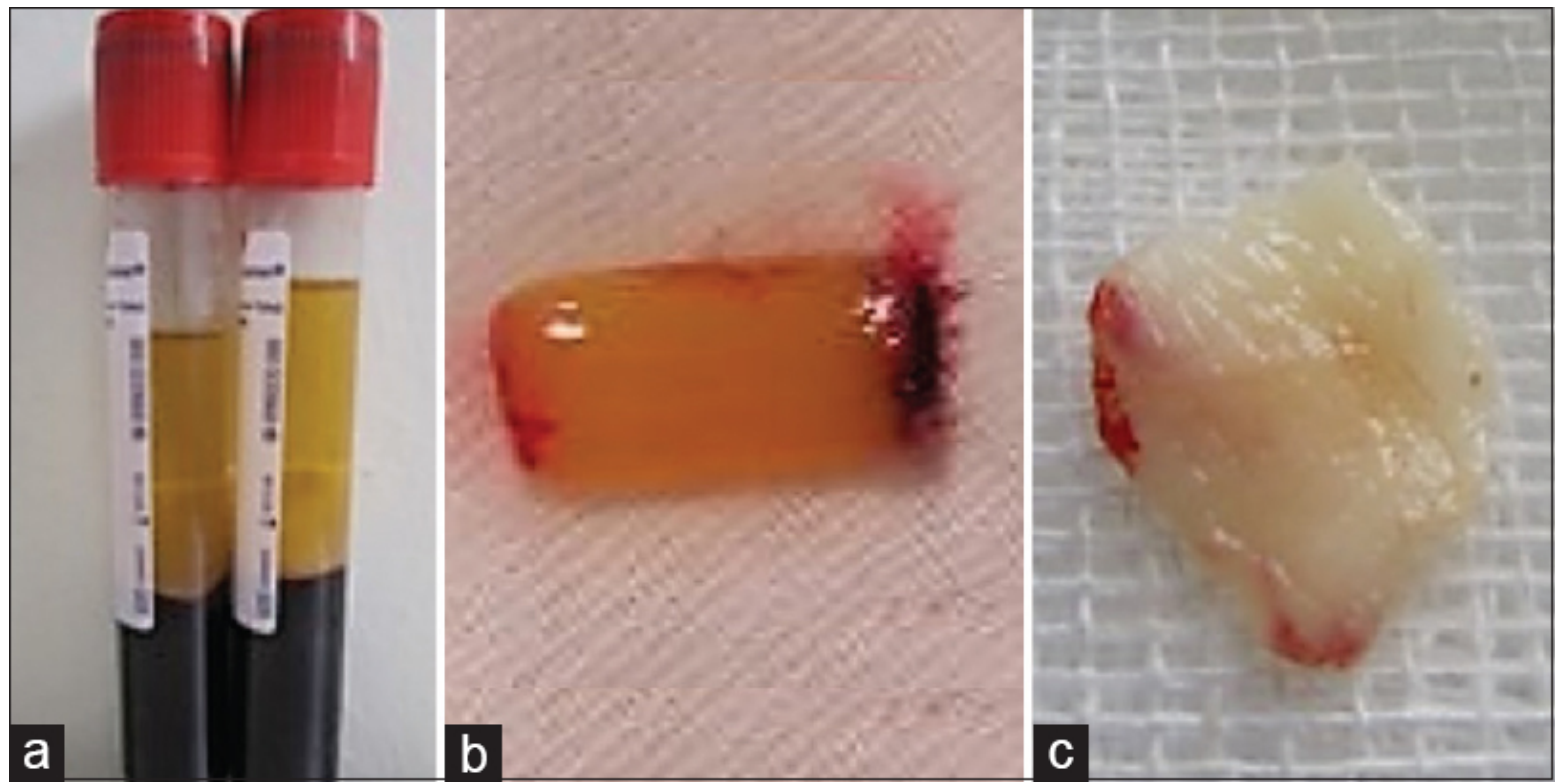

Figure 2. a) Structured fibrin clot in the middle of the tube b) PRF clot itself c) Autologous fibrin membrane after driving out the serum 


\subsection{The Mechanism of Action of Platelet Rich Fibrin}

The fundamental principle behind regeneration is based on hemostasis and tissue healing [2]. PRF uses its action from these principles and enables regeneration [10]. There are three possible mechanisms;

Mechanism 1: The fibrin matrix acts as a scaffold for recruitment and migration of cells like fibroblasts, endothelial and epithelial cells throughout the reparative process.

Mechanism 2: The components of the PRF i.e., platelets, leukocytes, neutrophils and monocytes secrete growth factors which facilitate the recruitment of the cells that aid in regeneration and wound healing.

Mechanism 3: Fibrin matrix mediates neoangiogenesis and the secretion of growth factors like vascular endothelial growth factor (VEGF). The new blood vessels bring in the essential nutrients required for sustaining cell metabolism and regeneration.

\subsection{Clinical Application of Platelet Rich Fibrin in Periodontics}

It has been reported that PRF can stimulate cell proliferation of osteoblasts, gingival and periodontal ligament fibroblasts and can suppress the growth of oral epithelial cells, thereby facilitating periodontal regeneration [11]. It has found various applications in periodontics such as in the treatment of intrabony defects, furcation involvement, guided tissue regeneration, root coverage procedures and as an adjunct to bone grafts which aid in the new attachment process in the periodontium $[12,13,14,15,16]$. In addition, PRF can be used in ridge augmentation, sinus lift and ridge preservation following tooth extraction.

\subsection{In Vitro Studies}

Numerous in vitro studies have been carried out using the PRF to tap its potential in various clinical applications. A study done by Gassling et al 2010 [17] showed that PRF was superior to collagen membrane in periosteal tissue engineering. Dohan et al 2009 observed that PRF was able to significantly induce a continuous stimulation of cells such as human gingival fibroblasts, dermal prekeratinocytes, preadipocytes and maxillofacial osteoblasts [18]. Another study by $\mathrm{Li}$ et al demonstrated that PRF improved periodontal regeneration and enhanced alveolar bone augmentation [11].

\section{Animal Studies}

Studies have been carried out using the autologous platelet concentrates using various animal models. In a porcine model Yang et al (2012) discovered that when dental bud cells were seeded into fibrin glue-PRF scaffold, it yielded a regenerated complete tooth structure. Recently, numerous studies have been conducted to test the potential of PRF around peri-implant defects in rabbit and dog models [19,20].
In animal study which was done by Sang-Hwa Lee et al, the effect of platelet-rich fibrin (PRF) on the osteogenesis and angiogenesis of rabbit calvarial defects was evaluated. The experimental site received PRF while the control sites were left filled with blood. They found that a significant linear increase of the area of newly formed osteoid on the defect margin in the PRF group. In addition, the intensity and percentage range of vascular endothelial growth factor (VEGF) expression showed a significant linear decrease trend with the time in the PRF group. According to this study, PRF may enhance the quality of bone regeneration [27].

Kökdere et al (2015) conducted an animal study to evaluate the efficiency of PRF and PRF/autogenous graft combination on bone healing. They found that either PRF used alone or in conjunction with autogenous bone graft, PRF accelerated the healing of the bone defects. There were statistically significant differences in osteoblast, osteoblast and new bone area values in PRF alone and autogenous graft with PRF than the other groups [28].

An animal study was conducted by Ocak H et al (2017) to compare the use of platelet-rich fibrin (PRF) and bovine-autogenous bone mixture for maxillary sinus floor elevation. A split-face model was used to apply 2 different filling materials for maxillary sinus floor elevation in 22 healthy adult sheep. In group 1 , bovine and autogenous bone mixture; and in group 2, PRF was used. The results of this study showed that bovine bone and autogenous bone mixture is superior to PRF as a grafting material in sinus-lifting procedures [34]. According to the results of this study, it may be suggested that PRF should not be used alone in sinus-lift procedure.

\subsection{Clinical Studies}

PRF membranes have been employed with bone graft materials to accelerate the healing process and this has been employed in maxillary sinus elevation procedures. Choukroun et al [21] found that PRF in combination with freeze-dried bone allograft (FDBA) significantly expedited the bone formation during sinus lift procedures. A new method of maxillary rehabilitation using PRF membranes, FDBA and $0.5 \%$ metronidazole displaced high degree of soft tissue esthetics and PRF showed to alleviate the postoperative complications like pain, edema and minor infections [22].

PRF can be used for ridge preservation after tooth extraction. Thakkar et al conducted a clinical trial to investigate clinically and radiographically, the bone fill in extraction sockets using demineralized freeze-dried bone allograft (DFDBA) alone and along with platelet-rich fibrin (PRF). They found that in both groups, there was significant reduction in loss of ridge width and ridge height However, when both the groups were compared the PRF group favored in the reduction of ridge width while there was no statistical difference in reduction of ridge height [23].

Pradeep and Sharma found that intrabony periodontal defects treated with PRF when compared to open flap debridement resulted in significant reduction in probing depth, gain in attachment level and greater bone fill [12]. Similar findings were observed by these researchers in 
mandibular Grade II furcation defect with PRF when compared to OFD [13].

Thorat $\mathrm{M}$ et al investigated the clinical and radiologic efficacy of autologous platelet-rich fibrin (PRF)+modified flap operation in the treatment of intrabony defects associated with localized aggressive periodontitis (LAP) compared to modified flap operation (MFO; Kirkland flap) alone. The results showed that the improvements in PD, CAL, and the radiographic bone fill in PRF treated sites compared to control sites were statistically significant [33].

In a split-mouth randomized clinical trial by Eren $\mathrm{G}$ et al, the clinical efficacy of platelet-rich fibrin (PRF) in combination with coronally advanced flap (CAF) in the treatment of localized gingival recessions was evaluated. Twenty-two patients with localized gingival recession defects (Miller I, II) participated in this split-mouth trial. Forty-four defects received either CAF plus PRF (test) or CAF with subepithelial connective tissue graft (SCTG) (control). The results showed that percentage of root coverage in test group was $92.7 \%$ and in control group was $94.2 \%$ and percentage of complete root coverage of the test and control groups was 72.7 and $77.3 \%$, respectively with there is no statistically significant difference between treatment groups in the terms of the width of keratinized tissue and the gingival thickness. According to the results of this study, PRF might be suggested as an alternative to the gold standard for treating gingival recession defects (SCTG) for the treatment of localized gingival recessions [29]. According to the clinical trial that was done by by Öncü et et (2017), The PRF in combination with a modified coronally advanced flap technique has the bonus advantage of being more comfortable during the postoperative period compared to a subepithelial connective tissue graft (SCTG) in combination with a modified coronally advanced flap (MCAF). In the same study, they found that localized gingival recessions could be successfully treated with MCAF plus PRF as well as MCAF plus SCTG [30]. However, in the systematic review which was conducted to assess the clinical efficacy of alternatives procedures including Platelet Rich Fibrin (PRF) compared to conventional procedures in the treatment of localized gingival recessions. They found that comparison between PRF and others technique was affected by large uncertainty and it was impossible to determine the differences between PRF and others technique [31].

Another clinical use of Platelet-Rich Fibrin (PRF) is the local application after third molar extraction. The benefits of applying PRF after tooth extraction are 1) Relieving pain. 2) Reliving post-operative swelling 3) Reducing the incidence of alveolar osteitis (AO). A meta-analysis was conducted by Hey Y et al (2017) to investigate the effect of local application of PRF on controlling postoperative signs and symptoms after the extraction of an impacted lower third molar. They found that PRF significantly relieves pain and 3-day postoperative swelling and reduces the incidence of alveolar osteitis AO [32].

In a recently published systematic review about the use of platelet-rich fibrin in regenerative dentistry, the systematic review showed that the use of PRF has been most investigated in periodontology for the treatment of periodontal intrabony defects and gingival recessions where the majority of studies have demonstrated favorable results in soft tissue management and repair. Little to no randomized clinical trials were found for extraction socket management although PRF has been shown to significantly decrease by tenfold dry sockets of third molars. Very little to no data was available directly investigating the effects of PRF on new bone formation in GBR, horizontal/vertical bone augmentation procedures, treatment of peri-implantitis, and sinus lifting procedures [35]. Future human randomized clinical studies evaluating the use of PRF on ridge augmentation thus remain necessary.

\section{Discussion}

It has long been opined that PRF trumps over PRP in various ways such as the easy handling and increased modulus of elasticity when compared to PRP. It also has been found that PRF expressed more of alkaline phosphatase that induced bone mineralization (24). Also, it has been observed in an in vitro study that PRF released more of the growth factors gradually with more durable effects on proliferation and differentiation of rat osteoblasts than PRP [24].

PRF has a long-standing association with the bone remodeling process [25]. RANK-RANKL interaction and activation from the bone forming cells results in the activation of the osteoclasts which induces bone resorption. Osteoprotegrin is a decoy receptor which nullifies the RANK-RANKL interaction and thereby improving bone formation. It has been demonstrated that PRF increases the expression of extracellular signal-regulated protein kinase (p-ERK) and osteoprotegrin (OPG) and thereby ascertaining its role in bone regeneration [25].

In the context of periodontal regeneration, it has been observed that PRF significantly stimulated the cellular proliferation of osteoblasts, gingival and periodontal ligament fibroblasts. It was also concluded that PRF had the potential to increase the attachment of osteoblasts, proliferation and simultaneously upregulating collagen production and these cumulative effects results in periodontal regeneration [26].

\section{Conclusion}

Autologous platelet concentrates have been targeted for their regenerative potential as it is obtained from the human blood and it can triumph over synthetic substitutes in their varied properties. The concept of obtaining a cellseeded matrix by drawing the patient's blood and centrifugation for 8-14 minutes is revolutionary in terms of clinical feasibility. It can be easily employed as a chairside procedure and it provides a local delivery of fibrin and enriched growth factors necessary for wound healing and regeneration. Future direction can be focused towards incorporating nanomaterials that allow for the incorporation of biomolecules which will significantly improve the efficacy of fibrin in various bone regenerative applications. 


\section{References}

[1] Mosesson MW, Siebenlist KR, Meh DA. The structure and biological features of fibrinogen and fibrin. Ann N Y Acad Sci. 2001; 936: 11-30.

[2] Clark RA. Fibrin and wound healing. Ann N Y Acad Sci. 2001; 936: 355-67.

[3] Collen A, Koolwijk P, Kroon M, van Hinsbergh VW. Influence of fibrin structure on the formation and maintenance of capillary-like tubules by human microvascular endothelial cells. Angiogenesis. 1998; 2(2): 153-65.

[4] Matras H. Fibrin sealant in maxillofacial surgery. Development and indications. A review of the past 12 years. Facial PlastSurg FPS. 1985; 2(4): 297-313.

[5] Chabbra S, Chabbra N, Vaid P. Platelet Concentrates: A New Alternative to Bone Regeneration. 2013 Jan 8; 2(2): 118-21.

[6] Marx RE, Carlson ER, Eichstaedt RM, Schimmele SR, Strauss JE, Georgeff KR. Platelet-rich plasma: Growth factor enhancement for bone grafts. Oral Surg Oral Med Oral Pathol Oral RadiolEndod. 1998 Jun; 85(6): 638-46.

[7] Dohan Ehrenfest DM, Rasmusson L, Albrektsson T. Classification of platelet concentrates: from pure platelet-rich plasma (P-PRP) to leucocyte- and platelet-rich fibrin (L-PRF). Trends Biotechnol. 2009 Mar; 27(3): 158-67.

[8] Dohan DM, Choukroun J, Diss A, Dohan SL, Dohan AJJ, Mouhyi $\mathrm{J}$, et al. Platelet-rich fibrin (PRF): a second-generation platelet concentrate. Part I: technological concepts and evolution. Oral Surg Oral Med Oral Pathol Oral RadiolEndod. 2006 Mar; 101(3): e37-44.

[9] Dhurat R, Sukesh M. Principles and Methods of Preparation of Platelet-Rich Plasma: A Review and Author's Perspective. J Cutan Aesthetic Surg. 2014 Dec; 7(4): 189-97.

[10] van Hinsbergh VW, Collen A, Koolwijk P. Role of fibrin matrix in angiogenesis. Ann N Y Acad Sci. 2001; 936: 426-37.

[11] Li Q, Pan S, Dangaria SJ, Gopinathan G, Kolokythas A, Chu S, et al. Platelet-rich fibrin promotes periodontal regeneration and enhances alveolar bone augmentation. BioMed Res Int. 2013; 2013: 638043 .

[12] Sharma A, Pradeep AR. Treatment of 3-wall intrabony defects in patients with chronic periodontitis with autologous platelet-rich fibrin: a randomized controlled clinical trial. J Periodontol. 2011 Dec; 82(12): 1705-12.

[13] Sharma A, Pradeep AR. Autologous platelet-rich fibrin in the treatment of mandibular degree II furcation defects: a randomized clinical trial. J Periodontol. 2011 Oct; 82(10): 1396-403.

[14] Anilkumar K, Geetha A, Umasudhakar null, Ramakrishnan T, Vijayalakshmi R, Pameela E. Platelet-rich-fibrin: A novel root coverage approach. J Indian SocPeriodontol. 2009 Jan; 13(1): 50-4.

[15] Ozdemir H, Ezirganli S, Isa Kara M, Mihmanli A, Baris E. Effects of platelet rich fibrin alone used with rigid titanium barrier. Arch Oral Biol. 2013 May; 58(5): 537-44.

[16] Shah M, Patel J, Dave D, Shah S. Comparative evaluation of platelet-rich fibrin with demineralized freeze-dried bone allograft in periodontal infrabony defects: A randomized controlled clinical study. J Indian SocPeriodontol. 2015 Feb; 19(1): 56-60.

[17] Gassling V, Douglas T, Warnke PH, Açil Y, Wiltfang J, Becker ST. Platelet-rich fibrin membranes as scaffolds for periosteal tissue engineering. Clin Oral Implants Res. 2010 May; 21(5): 543-9.

[18] Dohan Ehrenfest DM, Diss A, Odin G, Doglioli P, Hippolyte M-P, Charrier J-B. In vitro effects of Choukroun's PRF (platelet-rich fibrin) on human gingival fibroblasts, dermal prekeratinocytes, preadipocytes, and maxillofacial osteoblasts in primary cultures. Oral Surg Oral Med Oral Pathol Oral RadiolEndodontology. 2009 Sep; 108(3): 341-52.

[19] Neiva RF, Gil LF, Tovar N, Janal MN, Marao HF, Bonfante EA, et al. The Synergistic Effect of Leukocyte Platelet-Rich Fibrin and Micrometer/Nanometer Surface Texturing on Bone Healing around Immediately Placed Implants: An Experimental Study in Dogs. BioMed Res Int. 2016; 2016: 9507342.

[20] Şimşek S, Özeç İ, Kürkçü M, Benlidayı E. Histomorphometric Evaluation of Bone Formation in Peri-Implant Defects Treated
With Different Regeneration Techniques: An Experimental Study in a Rabbit Model. J Oral MaxillofacSurg Off J Am Assoc Oral Maxillofac Surg. 2016 Sep; 74(9): 1757-64.

[21] Choukroun J, Diss A, Simonpieri A, Girard M-O, Schoeffler C, Dohan SL, et al. Platelet-rich fibrin (PRF): a second-generation platelet concentrate. Part V: histologic evaluations of PRF effects on bone allograft maturation in sinus lift. Oral Surg Oral Med Oral Pathol Oral RadiolEndod. 2006 Mar; 101(3): 299-303.

[22] Simonpieri A, Del Corso M, Sammartino G, Dohan Ehrenfest DM The relevance of Choukroun's platelet-rich fibrin and metronidazole during complex maxillary rehabilitations using bone allograft. Part I: a new grafting protocol. Implant Dent. 2009 Apr; 18(2): 102-11.

[23] Thakkar DJ, Deshpande NC, Dave DH, Narayankar SD. A comparative evaluation of extraction socket preservation with demineralized freeze-dried bone allograft alone and along with platelet-rich fibrin: A clinical and radiographic study. Contemp Clin Dent. 2016 Sep; 7(3): 371-6.

[24] He L, Lin Y, Hu X, Zhang Y, Wu H. A comparative study of platelet-rich fibrin (PRF) and platelet-rich plasma (PRP) on the effect of proliferation and differentiation of rat osteoblasts in vitro. Oral Surg Oral Med Oral Pathol Oral RadiolEndod. 2009 Nov; 108(5): 707-13.

[25] Chang I-C, Tsai C-H, Chang Y-C. Platelet-rich fibrin modulates the expression of extracellular signal-regulated protein kinase and osteoprotegerin in human osteoblasts. J Biomed Mater Res A. 2010 Oct; 95(1): 327-32.

[26] Tsai C-H, Shen S, Zhao J, Chang Y-C. Platelet-rich fibrin modulates cell proliferation of human periodontally related cells in vitro. J Dent Sci. 6.

[27] Lee S. H., Kim S. W., Lee J. Il. \& Yoon H. J. The effect of platelet-rich fibrin on bone regeneration and angiogenesis in rabbit cranial defects. Tissue Eng. Regen. Med. 12, 362-370 (2015).

[28] Kökdere NN, Baykul T, Findik Y. The use of platelet-rich fibrin (PRF) and PRF-mixed particulated autogenous bone graft in the treatment of bone defects: An experimental and histomorphometrical study. Dental Research Journal. 2015; 12(5): 418-424.

[29] Eren G, Atilla G. Platelet-rich fibrin in the treatment of localized gingival recessions: a split-mouth randomized clinical trial. Clin Oral Investig. 2014 Nov; 18(8): 1941-8.

[30] Öncü E. The Use of Platelet-Rich Fibrin Versus Subepithelial Connective Tissue Graft in Treatment of Multiple Gingival Recessions: A Randomized Clinical Trial. Int J Periodontics Restorative Dent. 2017 Mar/Apr; 37(2): 265-271.

[31] Amine K, El Amrani Y, Chemlali S, Kissa J. Alternatives to connective tissue graft in the treatment of localized gingival recessions: A systematic review. J Stomatol Oral Maxillofac Surg. 2017 Sep 9. pii: S2468-7855(17)30154-4.

[32] He Y, Chen J, Huang Y, Pan Q, Nie M. Local Application of Platelet-Rich Fibrin During Lower Third Molar Extraction Improves Treatment Outcomes. J Oral Maxillofac Surg. 2017 Jun 8. pii: S0278-2391(17)30611-0.

[33] Thorat M, Baghele ON, S RP. Adjunctive Effect of Autologous Platelet-Rich Fibrin in the Treatment of Intrabony Defects in Localized Aggressive Periodontitis Patients: A Randomized Controlled Split-Mouth Clinical Trial. Int J Periodontics Restorative Dent. 2017 Nov/Dec; 37(6): e302-e309.

[34] Ocak H, Kutuk N, Demetoglu U, Balcioglu E, Ozdamar S, Alkan A. Comparison of Bovine Bone-Autogenic Bone Mixture Versus Platelet-Rich Fibrin for Maxillary Sinus Grafting: Histologic and Histomorphologic Study. J Oral Implantol. 2017 Jun; 43(3): 194-201.

[35] Miron RJ, Zucchelli G, Pikos MA, Salama M, Lee S, Guillemette V, Fujioka-Kobayashi M, Bishara M, Zhang Y, Wang HL, Chandad F, Nacopoulos C, Simonpieri A, Aalam AA, Felice P, Sammartino G, Ghanaati S, Hernandez MA, Choukroun J. Use of platelet-rich fibrin in regenerative dentistry: a systematic review. Clin Oral Investig. 2017 Jul; 21(6): 1913-1927.

[36] Saluja H, Dehane V, Mahindra U. Platelet-Rich fibrin: A second generation platelet concentrate and a new friend of oral and maxillofacial surgeons. Annals of Maxillofacial Surgery. 2011; 1(1): $53-57$ 